\title{
Inequalities for the Schatten p-norm $\mathbb{V}$
}

\author{
By
}

\author{
Fuad KittaneH* and Hideki Kosari***
}

\begin{abstract}
For positive operators $A, B$ on a Hilbert space $H$ and an operator monotone function $f$ on $(0, \infty)$, the following inequalities are proved:

(1) When $\lim _{s \rightarrow 0^{+}} f(s)=0,\|f(A)-f(B)\| \leqq f(\|A-B\|)$.

(2) If $A \geqq a \geqq 0$ and $B \geqq b \geqq 0$, then for any operator $X$ on $H$,

$$
\text { , } f(A) X-X f(B)\left\|{ }_{p} \leqq C(a, b)\right\| A X-X R \|_{p},
$$

where $1 \leqq p \leqq \infty$ and

$$
C(a, b)= \begin{cases}\frac{f(a)-f(b)}{a-b} & \text { if } a \neq b, \\ f^{\prime}(a) & \text { if } a=b .\end{cases}
$$

Other related inequalities for the Schatten $p$-norm are also considered.

1980 Mathematical Subject Classification Primary 47A30, 47B10; Secondary 82A15.

Key words and phrases Schatten $p$-norm, operator monotone function.
\end{abstract}

\section{§1. Introduction}

The following inequalities, due respectively to Powers and Stormer [11, Lemma 4.1] and van Hemmen and Ando [5, Proposition 4.1], are of special importance in mathematical physics.

Theorem $\mathbb{A}$ (Powers and Stormer) Let $A$ and $B$ be positive operators on a Hilbert space $H$. Then $\left\|A^{1 / 2}-B^{1 / 2}\right\|_{2}^{2} \leqslant\|A-B\|_{1}$, or equivalently $\|A-B\|_{2}^{2} \leqslant$ $\left\|A^{2}-B^{2}\right\|_{1}$.

Theorem $\mathbb{B}$ (van Hemmen and Ando)。 Let $A$ and $B$ be positive operators on a Hilbert space $H$ with $A+B \geqslant c>0$. Then for any operator-monotone function $f$ and any symmetric norm $\Phi$,

Communicated by H. Araki, December 3, 1986.

* Department of Mathematics, United Arab Emirates University, Al-Ain, United Arab Emirates

** Department of Mathematics, College of General Education, Kyushu University, Fukuoka 810 , Japan 


$$
\begin{aligned}
& \|f(A)-f(B)\|_{\Phi} \leqslant\left(\frac{f\left(\frac{c}{2}\right)-f(0)}{\frac{c}{2}}\right)\|A-B\|_{\Phi} . \quad \text { In particular, } \\
& \left\|A^{r}-B^{r}\right\|_{\Phi} \leqslant\left(\frac{2}{c}\right)^{1-r}\|A-B\|_{\Phi} \text { for } 0<r \leqslant 1
\end{aligned}
$$

Theorem $\mathrm{A}$ has been recently generalized by the first named author [9, Corollary 2] to a general Schatten $p$-norm $(1 \leqslant p \leqslant \infty)$ so that $\|A-B\|_{2 p}^{2} \leqslant \| A^{2}$ $-B^{2} \|_{p}$. It follows easily by induction that $\|A-B\|_{2^{n} p}^{2^{n}} \leqslant\left\|A^{2^{n}}-B^{2^{n}}\right\|_{p}$ for every positive integer $n$ and $1 \leqslant p \leqslant \infty$.

In section 2 (Theorem 2.3) we will give a related inequality (involving an operator monotone function) for the usual operator norm $(p=\infty)$.

As for Theorem B, a natural generalization would be a commutator version of this result. Assuming that either $A$ or $B$ is positive definite enables us to obtain a commutator version of Theorem $B$ with a presice estimate. This is considered in Section 3 (Theorem 3.1).

Throughout this paper (except in Theorem 2.4), the term operator means a bounded operator on a Hilbert space $H$, and $C_{p}, 1 \leqq p \leqq \infty$, denotes the Schatten $p$-class equipped with the associated $p$-norm $\|\cdot\|_{p} . \quad\left(\|\cdot\|_{\infty}\right.$ is the usual operator norm.)

The first version of the present article with a slightly weaker version of Theorem 2.3 was written by the first named author in the spring of 1986 . The second named author would like to mention that the only contribution by $\operatorname{him}$ (H.K) is Theorem 2.3 in its present form and Theorem 2.4.

\section{§ 2. On the Powers-Stormer Inequality}

One important consequence of the generalized Powers-Stormer inequality [9, Corollary 2] is the following result concerning the continuity of the map $T \rightarrow|T|$ from $C_{p}$ into itself, where $|T|=\left(T^{*} T\right)^{1 / 2}$.

Theorem 2.1. If $T$ and $S$ are operators on $H$, then $\||T|-|S|\|_{2 p} \leqslant \| T$ $+S\left\|_{2 p}^{1 / 2}\right\| T-S \|_{2 p}^{1 / 2}$ for $1 \leqslant p \leqslant \infty$.

Proof. Applying the generalized Powers-Stormer inequality to the positive operators $|T|$ and $|S|$, we see that

$\||T|-|S|\|_{2 p}^{2} \leqslant\left\||T|^{2}-|S|^{2}\right\|_{p}$. But 


$$
\begin{aligned}
& |T|^{2}-|S|^{2}=T^{*} T-S^{*} S=\frac{1}{2}(T-S)^{*}(T+S)+\frac{1}{2}(T+S)^{*}(T-S) 。 \\
& \text { Thus }\left\||T|^{2}-|S|^{2}\right\|_{p} \leqslant \frac{1}{2}\left\|(T-S)^{*}(T+S)\right\|_{p}+\frac{1}{2}\left\|(T+S)^{*}(T-S)\right\|_{p} \\
& \leqslant \frac{1}{2}\|T-S\|_{2 p}\|T+S\|_{2 p}+\frac{1}{2}\|T+S\|_{2 p}\|T-S\|_{2 p} \\
& =\|T-S\|_{2 p}\|T+S\|_{2 p} \text {. }
\end{aligned}
$$

Here we used Holder's inequality for $C_{p}$ [4]. And therefore $\||T|-|S|\|_{2 p}^{2} \leqslant$ $\|T+S\|_{2 p}\|T-S\|_{2 p}$ as required.

It has been shown in [7, Theorem 5] that if $A$ and $B$ are positive operators on $H$ such that $A+B \geqslant c \geqslant 0$, then $c\|A-B\|_{p} \leqslant\left\|A^{2}-B^{2}\right\|_{p}$ for $1 \leqslant p \leqslant \infty$ (see also proposition 3.2 in [5]). Using this inequality we now give another related continuity result.

\section{Theorem 2.2 If $T$ and $S$ are operators on $H$ such that} $|T|+|S| \geqslant c \geqslant 0$, then $c\||T|-|S|\|_{p} \leqslant\|T+S\|_{2 p}\|T-S\|_{2 p}$ for $1 \leqslant p \leqslant \infty$.

Proof. Applying Theorem 5 in [7] to the operators $|T|$ and $|S|$, the desired conclusion follows as in the proof of Theorem 2.1 above.

Remarks (1) The coefficients 1 and $c$ in Theorems 2.1 and 2.2 respectively are the best possible as illustrated by the two-dimensional example $T=\left[\begin{array}{ll}0 & 1 \\ 0 & 0\end{array}\right]$ and $S=\left[\begin{array}{ll}0 & 0 \\ 1 & 0\end{array}\right]$.

(2) It has been known that for operators $T$ and $S$ on $H$,

$$
\begin{aligned}
& \||T|-|S|\| \leqq \frac{2}{\pi}\|T-S\|\left(2+\log \frac{\|T\|+\|S\|}{\|T-S\|}\right) \quad \text { (see [6]), } \\
& \||T|-|S|\|_{2} \leqq \sqrt{2}\|T-S\|_{2} \quad \text { (see [1] and [8]), } \\
& \||T|-|S|\|_{1} \leq \sqrt{2}\|T+S\|_{1}^{1 / 2}\|T-S\|_{1}^{1 / 2} \quad \text { (see [2] and [10]). }
\end{aligned}
$$

Using elementary calculus, it can be shown that for non-negative real numbers $a$ and $b,\left|a^{r}-b^{r}\right| \geqslant|a-b|^{r}$ for $r \geqslant 1$ and $\left|a^{r}-b^{r}\right| \leqslant|a-b|^{r}$ for $0<r \leqslant 1$. Since these two numerical inequalities are equivalent, we will focus our attention on the second one. This inequality says that the function defined on $[0, \infty]$ by $f(s)=s^{r}$ is a Lipschitz function of order $r$. A non-commutative version of this inequality is sought. If $r=\frac{1}{2^{n}}$ for some positive integer $n$, then 
it follows from the generalized Powers-Stormer inequality $(p=\infty)$ that $\left\|A^{r}-B^{r}\right\|$ $\leqslant\|A-B\|^{r}$, whenever $A$ and $B$ are positive operators on $H$. Actually it remains valid for a general $r$ between 0 and 1 . Indeed we will present a more general result involving an operator monotone function.

Recall that a real-valued continuous function $f$ on $(0, \infty)$ is said to be operator monotone if, for any positive operators $A, B$, the relation $A \leqq B$ always implies $f(A) \leqq f(B)$. It is well-known [3] that such an $f$ has the unique integral representation

$$
f(s)=\alpha+\beta s-\int_{0}^{\infty}\left(\frac{1}{t+s}-\frac{t}{t^{2}+1}\right) d \nu(t),
$$

where $\alpha$ is real, $\beta \geqq 0$, and $\nu$ is a positive measure on $(0, \infty)$ satisfying

$$
\int_{0}^{\infty} \frac{d \nu(t)}{t^{2}+1}<\infty
$$

The most important examples of operator monotone functions are $\log s$ and $s^{r}(0<r \leqq 1)$ with integral representations

$$
\log s=-\frac{\sin (r \pi)}{\pi} \int_{0}^{\infty}\left(\frac{1}{t+s}-\frac{t}{t^{2}+1}\right) d t
$$

and for $0<r<1$

$$
s^{r}=\cos (r \pi / 2)-\frac{\sin (r \pi)}{\pi} \int_{0}^{\infty}\left(\frac{1}{t+s}-\frac{t}{t^{2}+1}\right) t^{r} d t
$$

Let us further assume $\lim _{s \rightarrow 0^{+}} f(s)=0$ (so let us set $f(0)=0$ ). It is easy to see that

$$
0=f(0)=\alpha-\int_{0}^{\infty}\left(\frac{1}{t}-\frac{t}{t^{2}+1}\right) d \nu(t)
$$

and

$$
\begin{aligned}
f(s) & =\beta s+\int_{0}^{\infty}\left(\frac{1}{t}-\frac{1}{t+s}\right) d \nu(t) \\
& =\beta s+\int_{0}^{\infty} \frac{s}{t+s} \frac{d \nu(t)}{t} .
\end{aligned}
$$

It is clear from this expression that $f$ is concave (actually operator concave).

Theorem 2.3. If $A, B$ are positive operators on a Hilbert space $H$, then for any operator monotone function $f$ with $f(0)=0$ we have 


$$
\|f(A)-f(B)\| \leqq f(\|A-B\|) .
$$

Proof. At first let us assume $A \geqq B \geqq 0$. Since $A=B+(A-B)$ with $A-B \geqq 0$, we get

$$
\begin{aligned}
& A \leqq B+\|A-B\| 1, \\
& f(A) \leqq f(B+\|A-B\| 1) .
\end{aligned}
$$

Since $B$ and $\|A-B\| 1$ commute and $f$ is concave (for positive scalars $a, b$, we have $f(a+b) \leqq f(a)+f(b))$, we get

$$
f(A) \leqq f(B)+f(\|A-B\|) 1,
$$

which implies that

$$
\begin{aligned}
& (0 \leqq) f(A)-f(B) \leqq f(\|A-B\|) 1, \\
& \|f(A)-f(B)\| \leqq\|f(\|A-B\|) 1\|=f(\|A-B\|)
\end{aligned}
$$

Before dealing with the general case, we prepare the following claim: If $X=X_{1}-X_{2}$ with $X=X^{*}$ and $X_{i} \geqq 0$, then $\left\|X_{+}\right\| \leqq\left\|X_{1}\right\|$ and $\left\|X_{-}\right\| \leqq\left\|X_{2}\right\|$, where $X=X_{+}-X_{-}$is the Jordan decomposition. In fact, let $p$ be the support projection of $X_{+}$. Then, $X=X_{1}-X_{2} \leqq X_{1}$ implies

$$
(0 \leqq) p X p=X_{+} \leqq p X_{1} p
$$

and

$$
\left\|X_{+}\right\| \leqq\left\|p X_{1} p\right\| \leqq\left\|X_{1}\right\|
$$

Repeating the same argument for $X_{-}$, we also get $\left\|X_{-}\right\| \leqq\left\|X_{2}\right\|$. Now let us assume $A, B \geqq 0$, and $A-B=(A-B)_{+}-(A-B)_{-}$be the Jordan decomposition. Since $A+(A-B)_{-}=B+(A-B)_{+}$, we get

$$
f(A)-f(B)=\left[f\left(B+(A-B)_{+}\right)-f(B)\right]-\left[f\left(A+(A-B)_{-}\right)-f(A)\right] .
$$

Here, both of $f\left(B+(A-B)_{+}\right)-f(B)$ and $f\left(A+(A-B)_{-}\right)-f(A)$ are positive because of the operator monotonicity of $f$. Hence the above claim and the first half of the proof show that

$$
\begin{aligned}
\left\|(f(A)-f(B))_{+}\right\| & \leqq\left\|f\left(B+(A-B)_{+}\right)-f(B)\right\| \\
& \leqq f\left(\left\|(A-B)_{+}\right\|\right), \\
\left\|((f A)-f(B))_{-}\right\| & \leqq\left\|f\left(A+(A-B)_{-}\right)-f(A)\right\| \\
& \leqq f\left(\left\|(A-B)_{-}\right\|\right) .
\end{aligned}
$$

Therefore, we estimate 


$$
\begin{aligned}
\|f(A)-f(B)\| & =\operatorname{Max}\left(\left\|(f(A)-f(B))_{+}\right\|,\left\|(f(A)-f(B))_{-}\right\|\right) \\
& \leqq \operatorname{Max}\left(f\left(\left\|(A-B)_{+}\right\|\right), f\left(\left\|(A-B)_{-}\right\|\right)\right) \\
& =f\left(\operatorname{Max}\left(\left\|(A-B)_{+}\right\|,\left\|(A-B)_{-}\right\|\right)\right) \\
& =f(\|A-B\|)
\end{aligned}
$$

For $p \neq \infty$, one cannot estimate $\|f(A)-f(B)\|_{p}$ in terms of $\|A-B\|_{p}$ (even if $B=0$ ), the reason being that the trace $\operatorname{Tr}$ on $B(H)$ satisfies $\operatorname{Tr}(1)=\infty$. For a finite von Neumann algebra, a situation is completely opposite, and we have

Theorem 2.4. Let $M$ be a finite von Neumann algebra with a normalized faithful trace $T r$. For positive operators $A, B$ in the non-commutative $L^{p}$-space $L^{p}(M ; T r), 1 \leqq p \leqq \infty$, and an operator monotone function $f$ with $f(0)=0$, we have

$$
\|f(A)-f(B)\|_{p} \leqq 2 f\left(\|A-B\|_{p}\right) .
$$

The definition of $L^{p}(M ; T r)$ and $\|\cdot\|_{p}$ can be found for example in [12], but the reader unfamiliar with von Neumann algebras may consider the following situation: Let $A, B$ be in $M_{n}(C)$, the set of the $n \times n$-matrices, and the Schatten $p$-norm on $M_{n}(C)$ is defined by using the normalized trace $(\operatorname{Tr}(A)$ $=n^{-1} \times \sum_{i=1}^{n} A_{i i}$ ).

Proof. Recall the expression

$$
f(s)=\beta s+\int_{0}^{\infty} \frac{s}{t+s} \frac{d \nu(t)}{t}
$$

before Theorem 2.3. Since

$$
f(A)-f(B)=\beta(A-B)+\int_{0}^{\infty}\left\{A(t+A)^{-1}-B(t+B)^{-1}\right\} \frac{d \nu(t)}{t},
$$

we get

$$
\|f(A)-f(B)\|_{p} \leqq \beta\|A-B\|_{p}+\int_{0}^{\infty}\left\|A(t+A)^{-1}-B(t+B)^{-1}\right\|_{p} \frac{d \nu(t)}{t} .
$$

Firstly, the well-known formula

$$
A(t+A)^{-1}-B(t+B)^{-1}=t(t+B)^{-1}(A-B)(t+A)^{-1}
$$

implies that

$$
\begin{aligned}
\left\|A(t+A)^{-1}-B(t+B)^{-1}\right\|_{p} & \leqq t\left\|(t+B)^{-1}\right\|\|A-B\|_{p}\left\|(t+A)^{-1}\right\| \\
& \leqq t^{-1}\|A-B\|_{p}
\end{aligned}
$$


Secondly, the obvious fact

$$
-1 \leqq-B(t+B)^{-1} \leqq A(t+A)^{-1}-B(t+B)^{-1} \leqq A(t+A)^{-1} \leqq 1
$$

and $\operatorname{Tr}(1)=1$ (hence $\|1\|_{p}=1$ ) imply that

$$
\left\|A(t+A)^{-1}-B(t+B)^{-1}\right\|_{p} \leqq 1 .
$$

We thus have shown

$$
\left\|A(t+A)^{-1}-B(t+B)^{-1}\right\|_{p} \leqq \operatorname{Min}\left(1, t^{-1}\|A-B\|_{p}\right) .
$$

It is elementary to see that the above right side is majorized by $2\|A-B\|_{p}(t+$ $\left.\|A-B\|_{p}\right)^{-1}$. Therefore, we get

$$
\|f(A)-f(B)\|_{p} \leqq \beta\|A-B\|_{p}+2 \int_{0}^{\infty}\|A-B\|_{p}\left(t+\|A-B\|_{p}\right)^{-1} \frac{d \nu(t)}{t},
$$

and the right side is obviously majorized by $2 f\left(\|A-B\|_{p}\right)$.

\section{§3. On the van Hemmen-Ando Inequality}

In this section we will establish a commutator version of the van HemmenAndo type inequality as stated in Theorem B. Although, our inequalities are valid for any symmetric norm on sequences, we will formulate these results in terms of the Schatten $p$-norm $(1 \leqslant p \leqslant \infty)$. Our technique is again the theory of operator-monotone functions.

Theorem 3.1. If $A$ and $B$ are positive operators on $H$ such that $A \geqslant a \geqslant 0$ and $B \geqslant b \geqslant 0$, then for any operator-monotone function $f$ and any operator $X$ on $H,\|f(A) X-X f(B)\|_{p} \leqslant C(a, b)\|A X-X B\|_{p}$, where

$$
C(a, b)=\left\{\begin{array}{cc}
\frac{f(a)-f(b)}{a-b} & \text { if } a \neq b \\
f^{\prime}(a) & \text { if } a=b
\end{array} \text { and } 1 \leqslant p \leqslant \infty\right.
$$

Proof. Let $f(s)=\alpha+\beta s-\int_{0}^{\infty}\left(\frac{1}{t+s}-\frac{t}{t^{2}+1}\right) d \nu(t)$ be the integral representation of $f$. Then

$$
f(A) X-X f(B)=\beta(A X-X B)+\int_{0}^{\infty}\left(X(B+t)^{-1}-(A+t)^{-1} X\right) d \nu(t) .
$$

Since $X(B+t)^{-1}-(A+t)^{-1} X=(A+t)^{-1}(A X-X B)(B+t)^{-1}$, it follows that $\|f(A) X-X f(B)\|_{p} \leqslant \beta\|A X-X B\|_{p}+\int_{0}^{\infty}\left\|(A+t)^{-1}(A X-X B)(B+t)^{-1}\right\|_{p} d \nu(t)$. 
But $\left\|(A+t)^{-1}(A X-X B)(B+t)^{-1}\right\|_{p} \leqslant\left\|(A+t)^{-1}\right\|\left\|(B+t)^{-1}\right\|\|A X-X B\|_{p}$

$$
\leqslant \frac{1}{(t+a)(t+b)}\|A X-X B\|_{p} .
$$

Therefore, $\|f(A) X-X f(B)\|_{p} \leqslant \beta\|A X-X B\|_{p}+\left(\int_{0}^{\infty} \frac{d \nu(t)}{(t+a)(t+b)}\right)\|A X-X B\|_{p}$

$$
=\left(\beta+\int_{0}^{\infty} \frac{d \nu(t)}{(t+a)(t+b)}\right)\|A X-X B\|_{p} \text {. }
$$

Now the proof can be completed by noticing that

$$
\beta+\int_{0}^{\infty} \frac{d \nu(t)}{(t+a)(t+b)}= \begin{cases}\frac{f(a)-f(b)}{a-b} & \text { if } a \neq b, \\ f^{\prime}(a) & \text { if } a=b .\end{cases}
$$

An important special case of Theorem 3.1, which deserves attention is the following result.

Corollary 3.2. If $A$ and $B$ are positive operators on $H$ such that $A \geqslant c>0$ and $B \geqslant c>0$, then for any operator $X$ on $H,\left\|A^{r} X-X B^{r}\right\|_{p} \leqslant r c^{r-1}\|A X-X B\|_{p}$, where $0<r \leqslant 1$ and $1 \leqslant p \leqslant \infty$.

In comparison with Theorem 5 in [7] or Proposition 3.2 in [5], it follows from Theorem 3.1 that if $A$ and $B$ are positive operators on $H$ such that $A \geqslant c$ $>0$ and $B \geqslant c>0$, then $2 c\|A-B\|_{p} \leqslant\left\|A^{2}-B^{2}\right\|_{p}$ for $1 \leqslant p \leqslant \infty$. Equality holds if and only if $A=B$ as demonstrated in the following theorem.

Theorem 3.3. Assume that $A$ and $B$ are positive operators on $H$ with $A \geqslant c$ $>0$ and $B \geqslant c>0$. Then:

(i) $2 c\|A-B\|=\left\|A^{2}-B^{2}\right\|$ if and only if $A=B$.

(ii) $2 c\|A-B\|_{p}=\left\|A^{2}-B^{2}\right\|_{p}<\infty$ for some $1 \leqslant p<\infty$, if and only if $A=B$.

Proof. Let $T=A-B$. Then $A^{2}-B^{2}=A T+T B$. If $2 c\|A-B\|=\left\|A^{2}-B^{2}\right\|$, then $2 c\|T\|=\|A T+T B\|$. We will show that $T=0$. Since $T$ is self-adjoint, there exists a sequence $\left\{f_{n}\right\}$ of unit vectors in $H$ such that $T f_{n}-t f_{n} \rightarrow 0$ as $n \rightarrow \infty$, where $|t|=\|T\|$.

Now

$$
\begin{aligned}
2 c|t| & =|| A T+T B \| \geqslant\left|\left((A T+T B) f_{n}, f_{n}\right)\right| \\
& =\left|\left((T-t) f_{n}, A f_{n}\right)+\left(B f_{n},(T-t) f_{n}\right)+t\left(A f_{n}, f_{n}\right)+t\left(B f_{n}, f_{n}\right)\right| \\
& \geqslant|t|\left(\left(A f_{n}, f_{n}\right)+\left(B f_{n}, f_{n}\right)\right)-\left|\left((T-t) f_{n}, A f_{n}\right)+\left(B f_{n},(T-t) f_{n}\right)\right| .
\end{aligned}
$$

If $\lim _{n \rightarrow \infty}\left(A f_{n}, f_{n}\right)>c$ or $\lim _{n \rightarrow \infty}\left(B f_{n}, f_{n}\right)>c$ (we consider subsequences if necessary), 
then in view of the fact that $\lim _{n \rightarrow \infty}(T-t) f_{n}=0$, it follows that $2 c|t|>2 c|t|$ which is absurd. Therefore $\lim _{n \rightarrow \infty}\left(A f_{n}, f_{n}\right)=c$ and $\lim _{n \rightarrow \infty}\left(B f_{n}, f_{n}\right)=c$ and so $t=\lim _{n \rightarrow \infty}\left(T f_{n}, f_{n}\right)$ $=\lim _{n \rightarrow \infty}\left(A f_{n}, f_{n}\right)-\lim _{n \rightarrow \infty}\left(B f_{n}, f_{n}\right)=0$. Hence $T=0$, which proves (i). Next assume that for some $1 \leqslant p<\infty$ we have $2 c\|A-B\|_{p}=\left\|A^{2}-B^{2}\right\|_{p}<\infty$. Then $2 c\|T\|_{p}$ $=\|A T+T B\|_{p}<\infty$. Since $T$ is a compact self-adjoint operator, it must be diagonalizable. Let $\left\{e_{n}\right\}$ be an orthonormal basis for $H$ such that $T e_{n}=t_{n} e_{n}$. Then

$$
\begin{aligned}
(2 c)^{p}\|T\|_{p}^{p}=\|A T+T B\|_{p}^{p} & \geqslant \sum_{n}\left|\left((A T+T B) e_{n}, e_{n}\right)\right|^{p} \\
& =\sum_{n}\left|t_{n}\left(\left(A e_{n}, e_{n}\right)+\left(B e_{n}, e_{n}\right)\right)\right|^{p}
\end{aligned}
$$

If $\left(A e_{n}, e_{n}\right)>c$ or $\left(B e_{n}, e_{n}\right)>c$ for some $n$, then $(2 c)^{p}\|T\|_{p}^{p}>(2 c)^{p}\|T\|_{p}^{p}$ which is also absurd. Thus $\left(A e_{n}, e_{n}\right)=\left(B e_{n}, e_{n}\right)=c$ for all $n$, and so $t_{n}=\left(T e_{n}, e_{n}\right)=0$ for all $n$, hence $T=0$, which proves (ii).

Applying Theorem 3.1 to the function $f(s)=\log s$, we see that if $A$ and $B$ are positive operators on $H$, then for any $c>0$ and any operator $X$ on $H, \| \log$ $(A+c) X-X \log (B+c)\left\|_{p} \leqslant \frac{1}{c}\right\| A X-X B \|_{p}$, where $1 \leqslant p \leqslant \infty$. In particular, $\|\log (A+c)-\log (B+c)\|_{p} \leqslant \frac{1}{c}\|A-B\|_{p}$. If we further require that $0<c<$ $\|A-B\|$, then we have the following inequality for the usual operator norm, the proof of which has a flavor similar to that of Theorem 2.4 .

Theorem 3.4 If $A$ and $B$ are positive operators on $H$ and $c$ is a constant such that $0<c<\|A-B\|$, then

$$
\|\log (A+c)-\log (B+c)\| \leqslant \log \left(\frac{e}{c}\|A-B\|\right) .
$$

Proof. Since $\log s=-\int_{0}^{\infty}\left(\frac{1}{t+s}-\frac{t}{t^{2}+1}\right) d t$, it follows that $\log (A+c)-\log$ $(B+c)=\int_{0}^{\infty}\left((B+c+t)^{-1}-(A+c+t)^{-1}\right) d t$. Now if $r>0$, we write

$$
\begin{aligned}
\log (A+c)-\log (B+c) & =\int_{0}^{r}\left((B+c+t)^{-1}-(A+c+t)^{-1}\right) d t \\
& +\int_{r}^{\infty}\left((B+c+t)^{-1}-(A+c+t)^{-1}\right) d t
\end{aligned}
$$

Thus

$$
\|\log (A+c)-\log (B+c)\| \leqslant \int_{0}^{r}\left\|(B+c+t)^{-1}-(A+c+t)^{-1}\right\| d t
$$




$$
+\int_{r}^{\infty}\left\|(B+c+t)^{-1}-(A+c+t)^{-1}\right\| d t
$$

To estimate the first integral on the right, we notice that

$$
\left\|(B+c+t)^{-1}-(A+c+t)^{-1}\right\| \leqslant \max \left(\left\|(B+c+t)^{-1}\right\|,\left\|(A+c+t)^{-1}\right\|\right) \leqslant \frac{1}{t+c}
$$

Hence $\int_{0}^{r}\left\|(B+c+t)^{-1}-(A+c+t)^{-1}\right\| d t \leqslant \int_{0}^{r} \frac{d t}{t+c}=\log \left(\frac{c+r}{c}\right)$. To estimate the second integral, we notice that

$$
\begin{aligned}
\left\|(B+c+t)^{-1}-(A+c+t)^{-1}\right\| & =\left\|(A+c+t)^{-1}(A-B)(B+c+t)^{-1}\right\| \\
& \leqslant \frac{1}{(t+c)^{2}}\|A-B\| .
\end{aligned}
$$

Hence $\int_{r}^{\infty}\left\|(A+c+t)^{-1}-(B+c+t)^{-1}\right\| d t \leqslant \int_{r}^{\infty} \frac{d t}{(t+c)^{2}}\|A-B\|=\frac{\|A-B\|}{c+r}$.

Therefore, $\|\log (A+c)-\log (B+c)\| \leqslant \log \left(\frac{c+r}{c}\right)+\frac{\|A-B\|}{c+r}$. But as a function of $r$, the expression $\log \left(\frac{c+r}{c}\right)+\frac{\|A-B\|}{c+r}$ attains its minimum at $r=$ $\|A-B\|-c$. Hence $\|\log (A+c)-\log (B+c)\| \leqslant \log \left(\frac{\|A-B\|}{c}\right)+1=\log \left(\frac{e}{c}\|A-B\|\right)$ as re-
quired.

\section{References}

[1] Araki H. and Yamagami, S., An inequality for the Hilbert-Schmidt norm, Commun. Math. Phys., 81 (1981), 89-96.

[2] Borchers, H. $C^{*}$-algebras and automorphism groups, Commun. Math. Phys., 88 (1983), 95-103.

[3] Donoghue, W.F., Monotone matrix functions and analysis ccntinuation, Springer Verlag, New York, 1974.

[4] Gohberg, I.C. and Krein, M.G., Introduction to the theory linear nonselfadjoint operators, Transl. Math. Monographs Vol. 18, Amer. Math. Soc., Providence, RI, 1969.

[5] van Hemmen ,J.L. and Ando, T., An inequality for trace ideals, Commun. Math. Phys., 76 (1980), 143-148.

[6] Kato, T., Continuity of the map $S \rightarrow|S|$ for linear operators, Proc. Japan Acad., 49 (1973), 157-160.

[7] Kittaneh, F., Inequalities for the Schatten p-norm II, to appear in Glasgow Math. J.

[8] - Inequalities for the Schatten p-norm III, Commun. Math. Phys., 104 (1986), 307-310.

[9] - Inequalities for the Schatten p-norm IV, Commun. Math. Phys., 106 (1986), 581-585. 
[10] Kosaki, H.. On the continuity of the map $\phi \rightarrow|\phi|$ from the predual of a $W^{*}$-algebra, J. Funct. Annal., 59 (1984), 123-131.

[11] Powers, R.T. and Stormer, E., Free states of the canonical anticommutation relations, Commun. Math. Phys., 16 (1970), 1-33.

[12] Segal, I., A non-commutative extension of abstract integration, Ann. Math., 57 (1953), 401-457. 
